A. Pramesh Rao, G. Swarup and Gopal-Krishna, eds.

\title{
The Luminosity Periodicity of Galaxies and Quasars in the Decametric Range
}

\author{
A.P. Miroshnichenko \\ Institute of Radio Astronomy, Krasnoznamyonnaya St.4, 310002, \\ Kharkov, Ukraine
}

\begin{abstract}
Estimations of the possible activity periods of galaxies and quasars were made from the UTR-2 catalogue samples by two methods.
\end{abstract}

If we assume that observed periodicity of the redshift distribution is caused by the recurrent nuclear activity, then a periodicity in the object luminosity distribution must also be seen. The results of our earlier work (Miroshnichenko 1994) favoured this assumption because the characteristic luminosity function evolution time was estimated to be less than the corresponding cosmological time.

To estimate the possible activity periods we used the statistically complete samples of 114 galaxies and 69 quasars, taken from from the UTR-2 catalogue at $25 \mathrm{MHz}$ in the declination zone $\delta=-13^{\circ}-+20^{\circ}$ for which optical data was available. The sample selection criteria for objects were: $S_{25} \geq 20 \mathrm{Jy}$, $m_{V} \leq 18.5^{m}$, where $S_{25}$ is the flux density at $25 \mathrm{MHz}, m_{V}$ the visual magnitude. The UTR-2 sensitivity for discrete sources $25 \mathrm{MHz}$ measurements is $10 \mathrm{Jy}$. The sample galaxies occupy the redshift range $z=0.002-0.881$, and the sample quasars occupy $z=0.129-2.975$ with mean value $\langle z\rangle \simeq 0.1$ for galaxies and $\langle z\rangle \simeq 1.0$ for quasars.

Let us the estimate of activity period as the difference $\Delta t_{L}$ of the corresponding cosmological times $t$ for the same luminosity objects from two subsamples: (1) for redshifts $z$ less than $\langle z\rangle$ and (2) for redshifts $z$ more than $\langle z\rangle$. The cosmological time calculations were carried out within the framework of Friedmann model (Zeldovich \& Novikov 1975) with $q_{0}=0.25$ and $H_{0}=100 \mathrm{~km}$ $\mathrm{s}^{-1} \mathrm{Mpc}^{-1}$. So, the simple activity period estimation is:

$$
\Delta t_{L}=t_{L}\left(z_{(1)}\right)-t_{L}\left(z_{(2)}\right)
$$

where $t_{L}\left(z_{(1)}\right), t_{L}\left(z_{(2)}\right)$ - the cosmological times for objects with same luminosity $L$ in our subsamples (1) and (2), respectively. These possible activity period values for sample galaxies and for sample quasars in the decametric and optical ranges are from $10^{8}$ to $10^{9}$ years.

We derived power spectra (periodograms) $P(\nu)$ (Deeming 1975) to find the periodicities in the sample object luminosity distribution with redshift. Our periodogram analysis revealed significant peaks (significance level is $Q=97$ $99 \%$ for the quasar luminosity distribution at redshifts $z_{p}=2.222 ; 1.124$ in both decametric and optical ranges, and at redshift $z_{p}=0.448$ in optical. Besides, we found the periodicity peak of optical luminosity at $z_{p}=0.224(Q=93.5 \%)$. The galaxy sample luminosity peaks were determined at $z_{p}=0.200(Q=98 \%)$ in the decametric range and at $z_{p}=0.0125(Q=90.5 \%)$ in optical. The subsample 
of powerful radio galaxies (FR II type) revealed peaks at $z_{p}=0.192(Q=91 \%)$ in the decametric range, and at $z_{p}=0.048(Q=99 \%)$ in optical.

The value $z_{p}=0.048$ agrees with result of Broadhurst et al.(1990) for the galaxy redshifts; the value $z_{p}=0.0125$, determined in our galaxy optical luminosity distribution, is close to the value shown by Duari, Gupta, \& Narlikar(1992) for quasar redshift distribution. The peak position at $z_{p}=2.222$ for the quasar luminosity distribution in decametric and optical ranges is in agreement with result by Gwyn \& Hartwick(1996) for the galaxy redshift distribution.

Thus, the redshift values, corresponding to periodicity peaks in the quasar and galaxy luminosity distributions in the decametric and optical ranges, are from $z_{p}=0.0125$ to $z_{p}=2.222$. Let us the distinguished values $z_{p}$ are multiple to smaller from those $\left(z_{p}=0.0125\right)$. Then we determine the corresponding space scale using formulae for distance $r$ in the comoving volume. For a Friedmann universe with $q_{0}=0.25$ and $H_{0}=100 \mathrm{~km} \mathrm{~s}^{-1} \mathrm{Mpc}^{-1}$, we find that the characteristic size in the object space distribution is $r_{p}=40 \mathrm{Mpc}$. Note, this value of $r_{p}$ corresponds to characteristic size of the galaxy superclusters.

In accordance with the new results in the fractal analysis of redshift surveys (Amendola \& Palladino 1999; Joyce, Montuori, \& Sylos Labini 1999) the galaxy clustering is described well by the fractal distribution with size $D=2$ on the scale 20-30 Mpc. Our estimation of the characteristic size in the object space distribution is close to other authors data.

We determined the time connected with luminosity periodicity from expression

$$
t-t_{0}=-\frac{1}{H_{0}}\left[z-\left(1.5+0.5 q_{0}\right) z^{2}\right]
$$

where $t, t_{0}$ are the cosmological time for the epoch $z$ and the present epoch, respectively (Zeldovich \& Novikov 1975). We obtain $t-t_{0}=1.2 \times 10^{8}$ years for $z_{p}=0.0125$ ( with $q_{0}=0.25$ and $H_{0}=100 \mathrm{~km} \mathrm{~s}^{-1} \mathrm{Mpc}^{-1}$ ) giving $4.3 \times 10^{8}$ years for $z_{p}=0.048$ and $1.4 \times 10^{9}$ years for $z_{p}=0.224$. So, our estimations of $t-t_{0}$ are in keeping with our first estimations $\Delta t_{L}$ in the decametric and optical ranges.

Thus, the cosmological periodicity in the sample luminosity distributions of galaxies and quasars may be the indicator of their nuclear activity recurrence.

\section{References}

Amendola, L., \& Palladino, E. 1999 ApJ, 514, L1

Broadhurst, T.,Ellis, R., Koo, D., \& Szalay, A. 1990 Nature, 343, 726

Deeming, T. 1975 Ap \&SS, 36, 137

Duari, D., Gupta, P., \& Narlikar,J. 1992 ApJ, 384, 35

Gwyn, S., \& Hartwick, F. 1996 ApJ, 468, L77

Joyce, M., Montuori, M., \& Sylos Labini, F. 1999 ApJ, 514, L5

Miroshnichenko, A.P. 1994 Cinematics \& Physics of Celestial Bodies, 10, 52

Zeldovich, Ya.B., \& Novikov, I.D. 1975 The Structure and the Evolution of the Universe (Moscow: Nauka) 